\title{
Comparative Analysis of Area Coverage in WSNs Using Various Grid-Based Node Deployment Schemes
}

\author{
P. Saritha Hepsibha and G. Sanjiv Rao
}

\begin{abstract}
In the last few years, there has been a tremendous interest on careful node deployment in wireless sensor networks, as it can be a very effective means for achieving the desired design goals. In this paper we show the implications of various grid based sensor node deployment schemes on network area coverage in WSNs. More generally, under some deployment assumptions, we present a simulation based results of area coverage for WSNs using grid deployment knowledge to motivate the use of this emerging paradigms. We also show the simulation of deployment schemes of WSNs using Network Simulator-2.34 and Mannasim.
\end{abstract}

Index Terms-Wireless sensor network, deployment knowledge, network coverage.

\section{INTRODUCTION}

A wireless sensor network (WSN) is a collection of small, low cost and battery powered devices called sensor nodes. The sensor nodes are also called as MICA2-DOT motes [1]. Each sensor node is capable of communicating with other sensor nodes wirelessly and monitoring an environment by sensing and gathering local data such as temperature, humidity, light or motion. These tiny sensor nodes with multi-functionalities make it possible to deploy wireless sensor networks (WSNs), which represent a significant improvement over traditional wired sensor networks [2].

A WSN is composed of densely deployed sensor nodes in a geographical area with a purpose of collecting and processing some useful data, and transmitting the data to nearby base stations for further processing. The transmission between the sensors is done by short range radio communications. The base station is assumed to be computationally well-equipped whereas the sensor nodes are resource- starved. A hierarchical wireless sensor network is explained in [3].

Usually the sensor networks are composed of one or more base stations and many sensor nodes scattered in a sensor field (i.e., deployment field or target field) as shown in Fig. 1. Each of these scattered sensor nodes has the capability to collect data and route data back to the base station. A base station or sink node serves as a gateway to another network, which is a powerful data processing unit, and also responsible for network management, collecting and performing costly operations on behalf of sensor nodes. As explained in a broadcast authentication scheme in WSNs [4], a base station acts like a commander, and broadcasts the commands to the sensors, and upon receiving those

Manuscript received December 19, 2012; revised February 25, 2013

The authors are with Dept of Information Technology, Sri Sai Aditya Institute of Science \& Technology, Suramplem, AP, India (e-mail: sarithahepsibha@yahoo.co.in). commands, sensors send results back to the base station.

To route data back to the base station, sensor nodes use a broadcast or multi-hop communication paradigm instead of point-to-point communications [5]. The base station may communicate with the task manager node via Internet or Satellite.

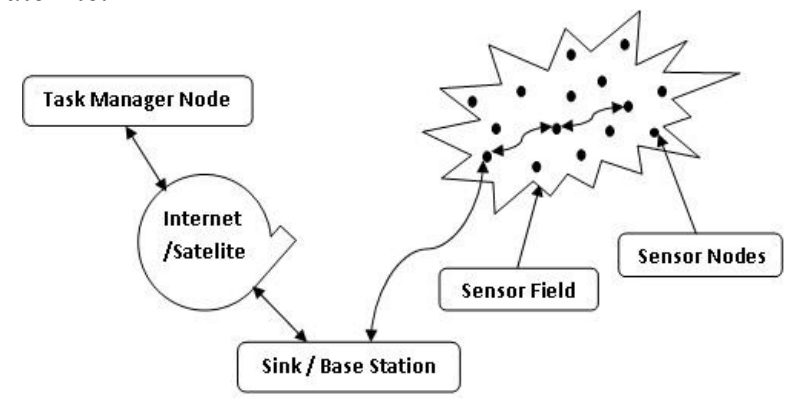

Fig. 1. Sensor nodes scattered in a target field

Using different sensors, WSNs can be implemented in many application areas including, security, entertainment, health care monitoring, automation, industrial monitoring, public utilities, and asset management. To support these applications, WSNs require to exhibit ad hoc networking properties. Ad hoc network properties can be found in [6]. However, the sensor networks need to have some unique features and application specific requirements, which made them different from ad hoc networks as listed in [2].

WSN devices have severe resource constraints in terms of energy, computation, memory and require large number of nodes. For many applications, sensors networks are usually deployed in sensing area to collect some information, and exhibits frequent changes in its topology. Therefore, deploying sensor nodes to provide complete area coverage is a basic issue to be addressed. Three alternative deployment schemes have been proposed in [6]. Depending upon the application requirements, node deployment can be either, application specific deterministic deployment, or random deployment or grid based deployment. Deterministic deployment is suitable for small-scale applications, where the sensors are placed directly in the required region. For large-scale applications, random deployment is suitable, where sensors are thrown randomly to form a network. Grid-based deployment is suitable for moderate to largescale applications, where individual sensors are placed exactly at grid points. In this paper, we make a comparison on the amount of network coverage for various grid-based node deployment strategies namely, square-grid, rectangular-grid, triangular-grid, and hexagonal-grid.

To demonstrate our approach more analytically, we have simulated the node deployment strategies using the simulation tools (such as Network Simulator-2.34 [7] and Mannasim [8]. 
The rest of this paper is organized as follows: Section II provides preliminary notions that are needed throughout the paper. Section III presents simulation based analysis of various grid-based node deployment schemes. Section IV focuses on comparative analysis of network area coverage using deployment knowledge and Section V concludes our work.

\section{PRELIMINARIES}

Our analysis will be based on some key results from the problem of measuring area of various geometric shapes inscribed in a two-dimensional circle.

\section{A. Assumptions \& Notations Used}

For instance, let the target region $T r$ to be deployed be in a circular shape. Therefore,

$$
\operatorname{Area}(T r)=\pi r^{2}
$$

Notice that here $r$ is the radius of the circular target region to be deployed. Now, we are interested in the calculation of maximum area coverage for various geometric shapes such as, square, rectangle, triangle and hexagonal shapes inscribed in a circle.

\section{B. Square Inscribed in a Circle}

If we consider a largest square inside a circle of radius $r$. Since, it is made up of four isosceles triangles whose equal sides are of length, $r$ and has area $1 / 2 r^{2}$.

Thus, the maximum coverage area of the contained square given by,

$$
\text { Area of a Square }=2 r^{2}
$$

\section{Rectangle Inscribed in a Circle}

Consider a rectangle with radius $r$ inscribed in a circle. Then, the radius of the circle is equal to the length of the hypotenuse of a right triangle which is duplicated 8 times in the rectangle. So, the area of the rectangle is 8 times the area of one of the right triangles. And,

The area of a right triangle $=1 / 2 r^{2} \cos \theta \sin \theta$. Here, $\theta$ is the angle at the triangle's corner that is located at the center of the circle. Therefore, the area of the whole rectangle can be,

$$
\text { Area of a Rectangle }=4 r^{2} \cos \theta \sin \theta
$$

\section{Triangle Inscribed in a Circle}

For instance, if we have an equilateral triangle inscribed in a circle, then, we know that in equilateral triangle all three sides are equal and all three internal angles are also equal and are each $60^{\circ}$. We also know,

Area of an equilateral triangle of side ' $a$ ' is, $a=\sqrt{3} a^{2} / 4$, and the radius of the circumscribed circle is, $r=\sqrt{3} a / 3$.

Therefore, Area of an equilateral triangle inscribed in a circle.

$$
\text { Area of a Triangle }=3 \sqrt{3} r^{2} / 4
$$

\section{E. Hexagonal Inscribed in a Circle}

Let us consider a hexagon is inscribed in a circle of radius ' $r$ '. Assuming it is a regular hexagon, there are 6 equilateral triangles with, sides of ' $r$ '. Thus, side of a hexagon $=r$. Now,

The area of each triangle $=$ base $\times$ height $/ 2$

$$
\begin{aligned}
& =\text { side } \times \text { apothem } / 2 \\
& =r \times \text { apothem } / 2
\end{aligned}
$$

Since, in equilateral triangle side $=r$

To get the area of the whole hexagon, just add up the areas of all the little triangles, thus,

$$
\begin{aligned}
\text { Area of Hexagon }= & \text { perimeter } \times \text { apothem } / 2 \\
& =6 \times r \times \text { apothem } / 2
\end{aligned}
$$

Since,

$$
\text { perimeter }=6 \times r \text {, and apothem }=\sqrt{r^{2}-(r / 2)^{2}}
$$

Therefore,

$$
\text { Area of a Hexagon }=6 \times r \times \sqrt{r^{2}-(r / 2)^{2}} / 2
$$

\section{F. Simulation Tools Used}

NS-2.34 is a discrete event network simulator that has been developed in 1989 as a variant of the REAL network simulator. Implementation and simulation of a network using NS-2 consists of 4 steps (1) Implementing the protocol by adding a combination of $\mathrm{C}++$ and OTcl code to NS-2's source base (2) Describing the simulation in an OTcl script (3) Running the simulation and (4) Analyzing the generated trace files. NS-2 is a powerful tool for simulating ad-hoc networks. But to simulate WSNs in NS-2, we need to have an additional module namely mannasim to represent the protocols that are specific to WSNs. MANNASIM is a framework for WSNs simulation, and is based on NS-2. It introduces new modules for design, development and analysis of different WSN applications. An excellent survey on simulation tools is given in [2]. WSN simulations with mannasim for various deployment schemes have been explained in [9].

\section{GRID BASED Node DEPLOYMENT SCHEMES}

In [6], it has state that grid deployment is an attractive approach for moderate to large-scale coverage-oriented deployment due to its simplicity and scalability. Based on these considerations, we focus on popular grid layouts such as rectangular-grid, square-grid, triangular-grid, and hexagonal-grid. For the coverage performance, grid-based deployment schemes are considered as a good deployment schemes in WSNs.

\section{A. Square Grid}

As described in [6], we investigate a square grid because of its natural placement strategy over a unit square. In the below Fig. 2 we present the simulation results of a square grid deployment of 9 sensor nodes. We calculated the 
approximate length and area of a unit square by using the formula given in [6].

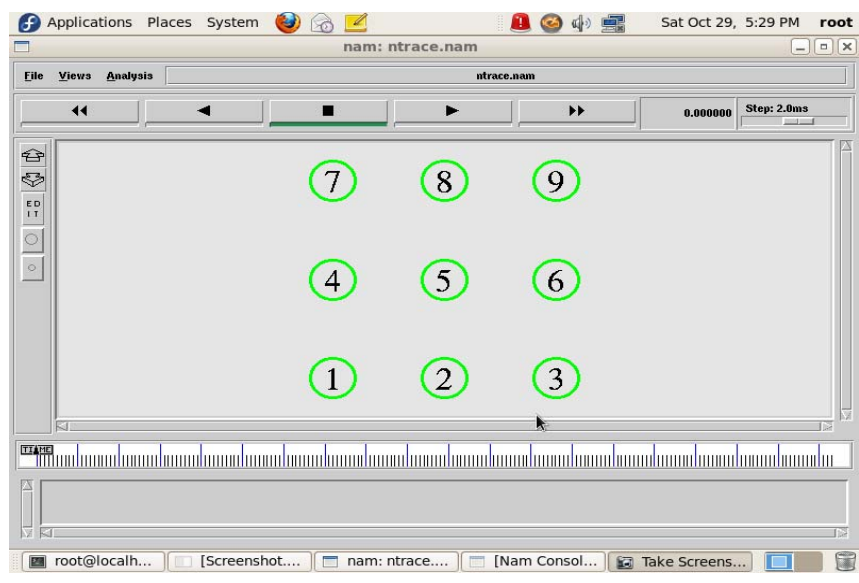

Fig. 2. Simulation of WSN using Square Grid Deployment

\section{B. Rectangular Grid}

As shown in the below Fig. 3. It visualizes a simulated sensor network with 20 nodes deployed with rectangular grid deployment scheme. The desired distance between consecutive droppings is achieved by controlling the time intervals. However, due to placement errors, this ideal deployment is not realistic.

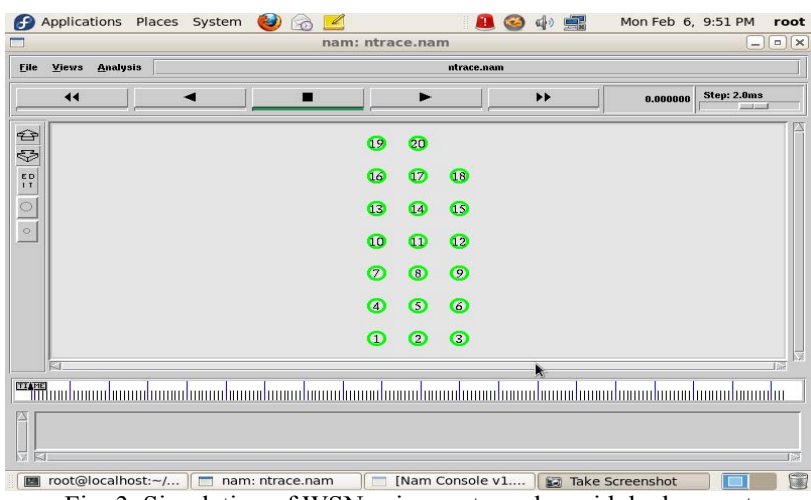

Fig. 3. Simulation of WSN using rectangular grid deployment

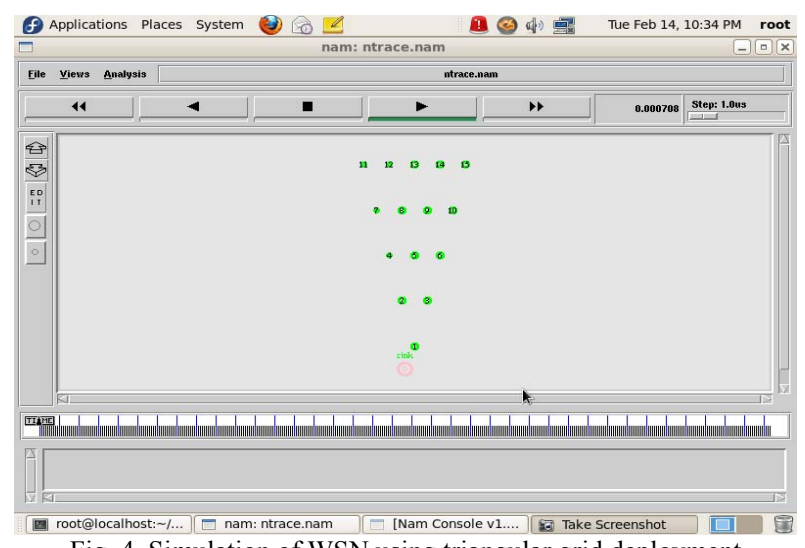

Fig. 4. Simulation of WSN using triangular grid deployment

\section{Triangular Grid}

As explained in [10], the triangular deployment scheme can cover a sensing area fully. It divides the target area into cells. Each cell represents the triangular-shaped sensing area. In the below Fig. 4, we presented the simulation-based result of a triangular deployment scheme. The below figure visualizes triangle deployment of 16 nodes among which node 0 represents sink node.

\section{Hexagonal Grid}

In this paper, we assume that the wireless sensor networks are made up of mica 2 motes and have an omni directional antenna. Therefore, each node has a round shape of communication area. One of the main goals of our work is to improve the covering area of interest to guarantee network coverage. We assumed a semi-regular tiling which uses triangle and hexagon in the two dimensional plane instead of considering a regular polygon which has the same side lengths and interior angle as described in [11]. As specified in [12], a tiling or tessellation of the plane is a process which uses a collection of polygons called tiles to cover the plane without gaps or overlaps. The below Fig. 5, it shows, a hexagonal grid deployment simulation, in which a target field is partitioned into hexagonal grids. The below figure visualizes the simulation of 24 nodes in the target area using hexagonal grid deployment to improve the network coverage.

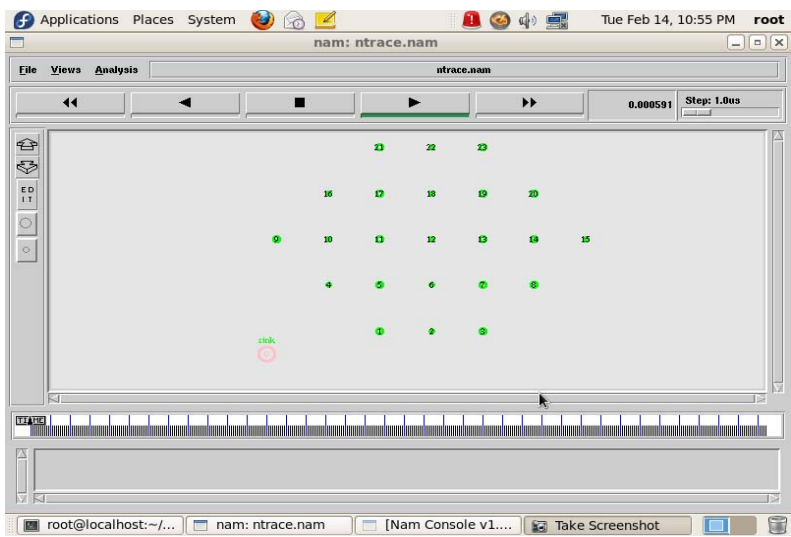

Fig. 5. Simulation of WSN using hexagonal grid deployment

\section{RELATED WORK}

In this section we present the possible network area coverage in WSNs, for various grid-based deployments schemes. Instead of deploying the nodes in a nondeterministic model (i.e. random deployment[13],[14]), we study the case were we partition the deployment region into various grids and analyze the maximum area coverage for various grid-based deployment schemes based on the key results from the problem of measuring area of various geometric shapes inscribed in a two-dimensional circle.

\section{A. Analysis of Area Coverage}

In our analysis, we assume the region the wireless network is deployed to be a circle of radius, $r$.

For instance, let the area of the circular region to be deployed, be 20,000sqmts .

Also, we know the area of a circle $=\pi r^{2}$.

$$
\begin{gathered}
20,000=\pi r^{2} \\
r^{2}=20,000 / \pi \\
r=\sqrt{20,000 / \pi} \\
r=79.79 m t s .
\end{gathered}
$$

Therefore, the radius of target region, $\operatorname{Tr}=79.79 \mathrm{mts}$. 


\section{B. Area Coverage using Square grid}

We are interested to calculate the maximum area coverage of sensor network while deploying the sensor nodes in square grid fashion within the target region of $79.79 \mathrm{mts}$ radius. From equation (2), area of a square inscribed in a circle of radius $r=2 r^{2}$

$$
=2 \times(79.79)^{2}
$$

Therefore, area of target region covered using square grid deployment $=12732.39$ sqmts .

\section{Area Coverage Using Rectangular Grid}

If we deploy the sensor nodes in rectangular grid fashion within the target region of $79.79 \mathrm{mts}$ radius, then from equation (3), the maximum area coverage can be obtained as, The area of the whole rectangle $=4 r^{2} \cos \theta \sin \theta$

$$
=4 \times(79.79)^{2} \times \cos 30^{\circ} \times \sin 30^{\circ}
$$

Here let the angle $\theta$ be $30^{\circ}$. Hence, maximum area of covered target region under rectangular grid deployment

$$
=11026.58 \text { sqmts } \text {. }
$$

\section{Area Coverage using Triangular Grid}

Now, if we consider the triangular grid deployment scheme over the target region, from equation (4), the maximum area covered by the best triangle inscribed in a circular target region with radius $79.79 \mathrm{mts}$ is given by,

$$
\begin{gathered}
\text { Area of the triangle }=3 \sqrt{3} r^{2} / 4 \\
=3 \times \sqrt{3} \times(79.79)^{2} / 4
\end{gathered}
$$

So, maximum target region covered under triangular grid deployment $=8269.93$ sqmis .

\section{E. Area Coverage using Hexagonal grid}

Now, we study the case, when we consider a hexagonal grid deployment scheme within the circular target region. From equation (5),

$$
\begin{aligned}
\text { Area of hexagon }=6 \times r \times \sqrt{r^{2}-(r / 2)^{2}} / 2 \\
=6 \times 79.79 \times \sqrt{(79.79)^{2}-(79.79 / 2)^{2}} / 2
\end{aligned}
$$

Therefore, maximum target region covered under hexagonal grid deployment $=16539.87$ sqmts .

\section{F. Comparative Analysis}

In order to deploy a sensor network with higher performance in terms of coverage, we have to gain the knowledge of various sensor node deployment schemes. As we discussed in the previous sections, the hexagonal grid exhibits higher network coverage than the remaining schemes. To make this point more clear, we assumed the area the wireless sensor network is deployed to be a circle of radius $r$. We also analyzed the maximum area coverage for various grid based deployment schemes by assuming basic geometric shapes inscribed in a circular shape target region of size 20,000sqmts, and depicted the simulation results in Fig. 6.and Fig. 7

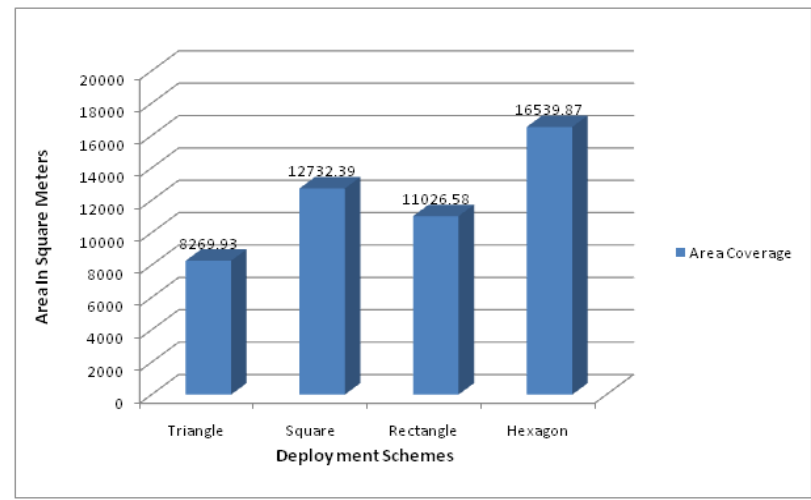

Fig. 6. Simulation of network area coverage WSN for various grid deployment schemes

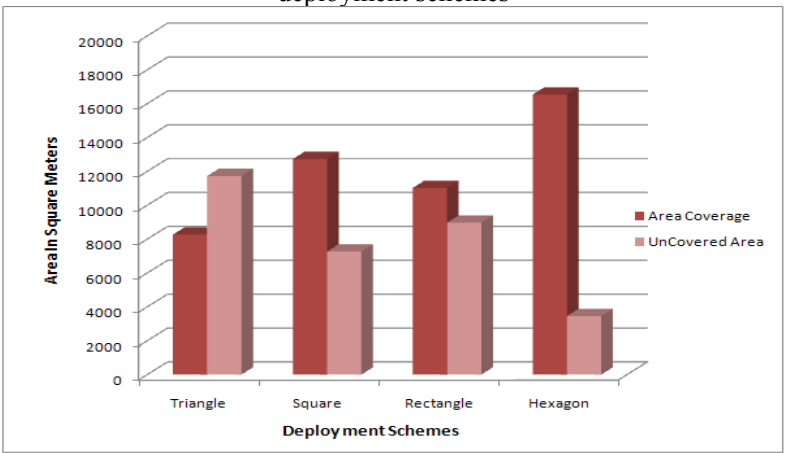

Fig. 7. Simulation of covered and uncovered areas in WSN for various grid deployment schemes

Here we calculated the maximum area coverage for each grid based deployment schemes such as, square, rectangular, triangular and hexagonal by considering different area values for circular target region to be deployed. From the above simulation results, it is shown that, among all deployment schemes, hexagonal grid deployment exhibits greater network coverage.

\section{CONCLUSION}

In this paper we focused on deployment knowledge to improve the network coverage. We studied various grid based node deployment schemes for WSNs. Under some deployment assumptions, we showed that the hexagonal grid deployment provides better area coverage than other schemes.

\section{ACKNOWLEDGMENT}

The authors would like to thank to Professor V. Valli Kumari, Dept of CS \& SE, Andhra University, Visakhapatnam and Sri Sai Aditya Institute of Science and Technology, Surampalem authorities for their constant support and cooperation.

\section{REFERENCES}

[1] Crossbow Technology Inc. [Online]. Available: http://www.xbow.com.

[2] E. E. Lopez, J. V. Alonso, A. S. M. Sala, P. P. Marino, and J. G. Haro, "Simulation Tools for Wireless Sensor Networks," presented at Summer Simulation Multi Conference - SPECTS 2005.

[3] A. K. Das, "Improving Identity-Based Random Key Establishment Scheme for Large-Scale Hierarchical Wireless Sensor Networks," International Journal of Network Security, vol. 13, no. 3, pp. 181-201, 2011.

[4] R. H. Wang, W. L. Du, and X. G. Liu, "ShortPK: A Short- Term Public Key Scheme for Broadcast Authentication in Sensor 
Networks," ACM Transactions on Sensor Networks, vol. 6, no. 1, 2009.

[5] I. F. Akyildiz, W. Su, and Y. Sankarasubramaniam, "A Survey on Sensor Networks," IEEE Communications Magazine, August 2002.

[6] Monica and A. K. Sharma, "Comparative Study of Energy Consumption for Wireless Networks Based on Random and Grid Deployment Strategies," International Journal of Computer Applications, vol. 6, no. 1, 2010.

[7] The Network Simulator, NS-2. [Online]. Available: http://www.isi.edu/nsnam/ns/

[8] Manna. [Online]. Available: http://www.mannasim.dcc.ufmg.br/

[9] G. S. V Rao and V. V. Kumari, "A Study on Deployment Schemes for Wireless Sensor Networks," Springer-LNICST, AUC 3.0, The Third International Workshop on Ad Hoc \& Ubiquitous Computing, 2012.

[10] Z. Yu and Y. Guan, "A Key Management Scheme Using Deployment Knowledge for Wireless Sensor Networks," IEEE Transactions on Parallel and Distributed Systems, vol. 19, no. 10, October 2008.

[11] C. H. Wu and Y. C. Chung, "A Tiling-Based Approach for Directional Sensor Network Deployment," in Proc. of IEEE Sensors 2010 Conference.

[12] B. Grunbaum and G. C. Shephard, "Tilings by Regular Polygons," Mathematics Magazine, vol. 50, no. 5, pp. 227-247, 1977.

[13] X. Y. Li, Y. J. Wang, W. S. Feng, M. Li, and Y. H. Liu, "Random Deployment of Wireless Sensor Networks: Power of Second Chance,"

in Proc. of the 15th International Computing and Combinatorics Conference, July 13-15, 2009. Niagara Falls, New York, U.S.A.

[14] W. Y. Poe and J. B. Schmitt, "Node Deployment in Large Wireless Sensor Networks: Coverage, Energy Consumption, and Worst-Case Delay," AINTEC'09, November 18-20, 2009, Bangkok, Thailand.

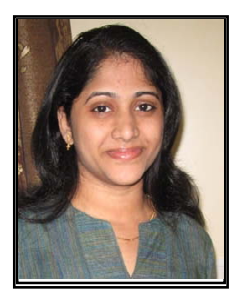

P. Saritha Hepsibha is currently working as assistant professor in Information Technology Department, Sri Sai Aditya Institute of Science \& Technology Surampalem, AP, and India. She received her M.Tech from Andhra University. Her research interests are in the areas of Security over Wireless Sensor Networks.

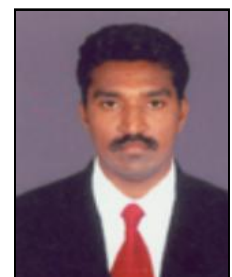

G. Sanjiv Rao is currently working as associate professor in Information Technology Department, Sri Sai Aditya Institute of Science \& Technology Surampalem, AP, India. $\mathrm{He}$ is working towards $\mathrm{Ph} . \mathrm{D}$. at College of Engineering, Andhra University. He received his M.Tech from the same institute. His research interests are in the areas of Security over Wireless Sensor Networks. He is a member of CSI. 